\title{
Subwavelength anti-diffracting beams propagating over more than 1,000 Rayleigh lengths
}

\author{
Eugenio DelRe ${ }^{1 \star}$, Fabrizio Di Mei ${ }^{12}$, Jacopo Parravicini1, Gianbattista Parravicini ${ }^{3}$, Aharon J. Agranat ${ }^{4}$ \\ and Claudio Conti ${ }^{1,5}$
}

Propagating light beams with widths down to and below the optical wavelength require bulky large-aperture lenses and remain focused only for micrometric distances ${ }^{1,2}$. Here, we report the observation of light beams that violate this localization/depth-of-focus law by shrinking as they propagate, allowing resolution to be maintained and increased over macroscopic propagation lengths. In nanodisordered ferroelectrics ${ }^{3,4}$ we observe a non-paraxial propagation of a sub-micrometresized beam for over 1,000 diffraction lengths, the narrowest visible beam reported to date ${ }^{5-8}$. This unprecedented effect is caused by the nonlinear response of a dipolar glass, which transforms the leading optical wave equation into a Klein-Gordon-type equation that describes a massive particle field ${ }^{9}$. Our findings open the way to high-resolution optics over large depths of focus, and a route to merging bulk optics into nanodevices.

As monochromatic light travels through a transparent material its optical field $\mathbf{E}$ is governed by the Helmholtz equation (HE) $\left(\nabla^{2}+n^{2} k_{0}^{2}\right) \mathbf{E}=0$, where $k_{0}=2 \pi / \lambda$ is the wavevector, $\lambda$ is the wavelength of the beam, and $n$ is the index of refraction ${ }^{10}$. The HE includes diffraction, which smears out the fine details of spatial information. In nanodisordered ferroelectrics, the photorefractive nonlinearity can cause light to obey a Klein-Gordon equation (KGE) $\quad\left(-\partial_{z^{\prime} z^{\prime}}^{2}+\nabla_{\perp}^{2}-n_{\mathrm{m}}^{2} k_{0}^{2}\right) \mathbf{E} \equiv\left(\square-n_{\mathrm{m}}^{2} k_{0}^{2}\right) \mathbf{E}=0$, which corresponds to a relativistic particle with mass given by the Einstein relation $m c^{2}=\hbar n_{\mathrm{m}} k_{0} c$ (see Methods).

In terms of propagation, a basic signature of the KGE regime is anti-diffraction; that is, diffraction-limited optical spots shrink instead of spreading. The simplest description of anti-diffraction can be formulated in the paraxial approximation, which predicts that

$$
\frac{w(z)}{w_{0}}=\sqrt{1-\frac{\lambda^{2}}{\pi^{2} n_{\mathrm{m}}^{2}(L) w_{0}^{4}} z^{2}}
$$

(see Supplementary Section 'Beam anti-diffraction law in the paraxial regime'), which connects the beam spot size $w(z)$ at a given distance $z$ along the propagation direction to the minimum spot size $w_{0}$ at $z=0$ through $\lambda$ and $n$ and the characteristic length scale $L^{11}$. For $L \ll \lambda$ the standard diffraction law ${ }^{12}$

$$
\frac{w(z)}{w_{0}}=\sqrt{1+\frac{\lambda^{2}}{\pi^{2} n^{2} w_{0}^{4}} z^{2}}
$$

holds, so for sufficiently large values of $z$, the beam has an angular spread that scales as $\Delta \theta \approx \lambda / n w_{0}$. In turn, for $L>\lambda$, equation (1) predicts beams that converge like funnels into a point-like focus at the critical value $z_{\mathrm{c}}=(n \pi / \lambda) w_{0}^{2}\left[(L / \lambda)^{2}-1\right]^{-1 / 2}$.

Anti-diffraction at $\lambda=633 \mathrm{~nm}$ was observed with the set-up presented in Fig. 1a. To achieve values of $L / \lambda>1$ we applied a rapid change in temperature to the crystal before launching the propagating light (see description of thermal shocks in the Methods). The $L \ll \lambda$ (diffraction) and the $L>\lambda$ (anti-diffraction) cases are shown in Fig. $1 b$ and $c$, respectively. In Fig. $1 b$, a round Gaussian beam is focused to its diffraction-limited spot at the input of the sample and naturally diffracts through the sample when standard cooling is performed, a behaviour compatible with $L \ll \lambda$. In this case the beams obey the HE in its paraxial approximation and spread following the basic Gaussian beam law. If a thermal shock is enacted and the laser is switched on once the temperature shock has been completed (at $t=0$ ), not only does the beam not spread, it shrinks to below its diffraction-limited input spot (Fig. 1c). In Supplementary Fig. 4 we report how the KGE regime builds over time. Anti-diffraction is observed in a transient that lasts for $\sim 10 \mathrm{~s}$.

In the simplified paraxial case, both the anti-diffraction of equation(1) and the diffraction of equation(2) can be formally unified into a single description by considering the effective permittivity

$$
\epsilon_{\mathrm{m}}=\frac{\epsilon_{r}}{1-\left(\frac{L}{\lambda}\right)^{2}}
$$

which reduces to the standard definition of the relative dielectric permittivity at optical frequencies $\epsilon_{\mathrm{m}}=\epsilon_{r}=n^{2}\left(\mu=\mu_{0}\right)$ for $L \ll \lambda$. When $\epsilon_{\mathrm{m}}<0$ (the wave evolution follows a KGE), beams do not have an 'uncertainty' diffraction relation that fixes the minimum beam angular spread $\Delta \theta$ for a given beam size $w_{0}$ (ref. 13) and the Gaussian beam naturally converges (see Supplementary Section 'Naturally converging beams in the $\epsilon_{\mathrm{m}}<0$ regime'). In contrast to the linear response theory, $\epsilon_{\mathrm{m}}$ is not the real part of a complex response function, but is a product of photorefraction and nonlinear beam propagation and is therefore not accompanied by an equally dynamic absorption coefficient associated with an imaginary part of the response.

The relationship between diffraction, anti-diffraction, the value of $L / \lambda$, and $\epsilon_{\mathrm{m}}$ is presented in Fig. 2a. In Fig. 2b,c the diffraction/anti-diffraction phenomenology (of which Fig. $1 b$ and $c$ are examples) are depicted as a function of the different thermal trajectories $T(t)$, where the value of $L / \lambda$ is evaluated through equation (1) from measured output beam widths. The peak value of $L / \lambda$ depends on the thermal history of the dipolar glass and is transient, so when the appropriate shock is enacted, the KGE regime emerges, with its

'Physics Department, University of Rome Sapienza, Rome 00185, Italy. ${ }^{2}$ Center for Life Nano Science@Sapienza, Istituto Italiano di Tecnologia, Rome 00161, Italy. ${ }^{3}$ Physics Department, University of Pavia, Pavia 27100, Italy. ${ }^{4}$ Applied Physics Department, Hebrew University of Jerusalem, Jerusalem 91904 , Israel. ${ }^{5}$ Institute for Complex Systems, National Research Council (ISC-CNR), Via dei Taurini 19, Rome 00185, Italy. ^e-mail: eugenio.delre@uniroma1.it 


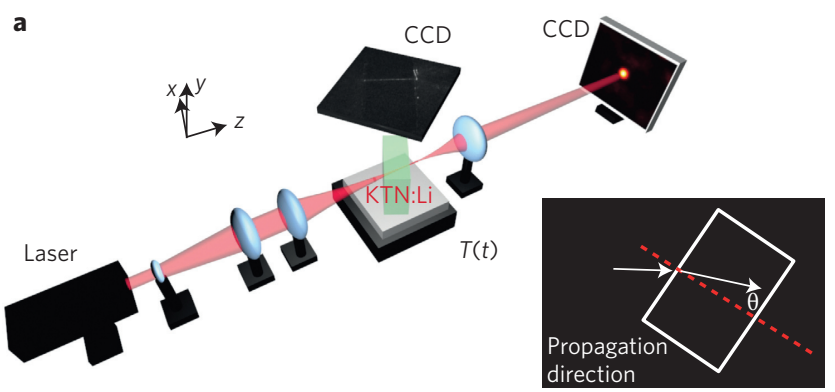

b No $T$ shock: diffraction $T(t)$
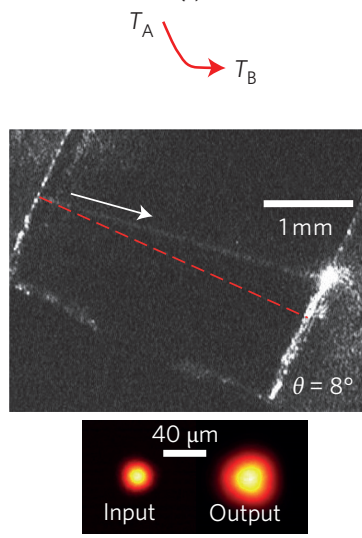

c T shock: anti-diffraction $T(t)$
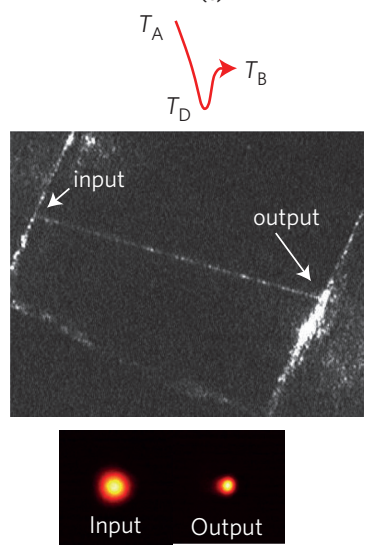

Figure 1 | Light beams that anti-diffract. a, Optical set-up to study the transition from the HE regime to the KGE regime (see Methods). b. Standard Gaussian diffraction for a laser beam focused to its minimum spot at the input facet of the crystal, with a peak intensity of $I_{\mathrm{r}}=1.4 \mathrm{~kW} \mathrm{~cm}^{-2}$, from a width of $11 \mu \mathrm{m}$ (FWHM of $13 \mu \mathrm{m}$ ) to $26 \mu \mathrm{m}$. c, Anti-diffraction after a thermal shock $T(t)$ (see Methods). Although the beam has its minimum spot size at the input, it shrinks below this limit, down to $7 \mu \mathrm{m}$ (the snapshot is at $t=5 \mathrm{~s}$ ), as it propagates. Note that beams anti-diffract independent of the propagation angle, in contrast to periodic systems and metamaterials (for example, $\theta=8^{\circ}$ and $17^{\circ}$ inside the crystal in $\mathbf{b}$ and in Supplementary Fig. 4, respectively). Top-view images capture stray scattered light and have an inherently low resolution $(\sim 2 \mu \mathrm{m})$.

negative effective permittivity calculated from equation (3) (Fig. 2d). Corresponding measured values of the output beam width relative to the input beam width are presented in Fig. 2e. The strong susceptibility and its associated large value of $L / \lambda$ appear only if the thermal shocks are larger than a threshold amplitude $\Delta T$, whereas the phenomenon is independent of the dwell time $\Delta t$. These traits indicate that the thermal shock activates a response typical of glassy systems such as polymers ${ }^{14}$ (Supplementary Fig. 5).

With long enough propagation (as $z_{\mathrm{c}}$ is approached), the original paraxial beam should eventually shrink and become non-paraxial. To explore the validity of the KGE predictions in the non-paraxial regime, we launch a laser beam exiting a saturated high-aperture microscope objective (Fig. 3). Figure 3 a shows the knife-edge super-resolution set-up used to detect the non-paraxial beam width (see Methods). After the thermal shock, a non-paraxial propagation emerges, with an ultra-tight-input $w_{0}=1.5 \mu \mathrm{m}$ spot (Fig. 3b) shrinking to $1.3 \mu \mathrm{m}$ after $3 \mathrm{~mm}$ propagation (Fig. 3c), equivalent to 134 diffraction (Rayleigh) lengths. When no thermal shock is enacted, the beam engulfs the entire sample, illuminating it (Fig. 3d). In the anti-diffracting case we observe the narrowest propagating visible light beam reported to date. To violate the limits of diffractive optics on spot size during propagation, we experimented with even narrower input launch beams using a fully illuminated long-working-distance dry objective (Edmund Optics, $\times 100, \mathrm{NA}=0.8$ ). As reported in Fig. 4, the subwavelength beam input of $0.49 \mu \mathrm{m}$ shrinks to $0.28 \mu \mathrm{m}$ as it leaves the sample output facet, below the $\lambda / 2=0.633 / 2=0.32 \mu \mathrm{m}$ limit, but still above the limit where the KGE breaks down at $w_{0} \simeq 0.1 \mu \mathrm{m}$, for $L / \lambda \simeq 1.1$ (see equation (7)). For the $L_{z} \simeq 3 \mathrm{~mm}$ distance, $w_{0}=0.49 \mu \mathrm{m}$ and $n_{0}=2.2$ at $\lambda=0.633 \mu \mathrm{m}$, corresponding to $\sim 1,150$ Rayleigh lengths of propagation, where the Rayleigh length $L_{\mathrm{D}}=n_{0} w_{0}^{2} \pi / \lambda \simeq 2.62 \mu \mathrm{m}$.

The transition from the HE regime to the KGE regime, activated by the thermal shock through the photorefraction diffusive response, leads to intensity-independent changes in the optical propagation, as reported in Supplementary Fig. 6. This indicates that the thermal effects for the investigated beam powers ( $P=0.1-2.2 \mathrm{~mW}$, measured before the launch lens) are negligible, and that the beams are not solitons, because they do not obey an intensity/width scaling $\mathrm{law}^{5}$. In fact, the beams obey an approximate KGE that is (1) linear, (2) foresees anti-diffraction as opposed to propagation invariance, and (3) is also valid in the non-paraxial regime, where the standard optical soliton model based on the generalized nonlinear Schrödinger equation fails. The dipolar processes that intervene in the ultra-tight beam propagation are investigated in Supplementary Fig. 7, which also reports time dynamics in the non-paraxial case.

The breaking of conventional diffraction limits can be intuitively understood in terms of the transition from the HE to the KGE. In the HE, expanding the optical electric field in its transverse Fourier components $\mathbf{E}=\int_{k_{x}, k_{y}} \mathrm{~d} k_{x} \mathrm{~d} k_{y} \mathbf{E}\left(k_{x}, k_{y}\right) \exp \left(\mathrm{i} k_{z} z+\mathrm{i} k_{x} x+\mathrm{i} k_{y} y\right)$, the propagating waves are bound to obey the relation $k_{x}^{2}+k_{y}^{2} \leq k_{0}^{2}$, so $\lambda / 2$ constitutes the lower limit to spatial resolution (in air). In turn, this limit is absent in the KGE regime, where the relative sign of the Laplacian term flips.

Previous studies in nanodisordered ferroelectrics have led to scale-free optics, a regime in which light cannot diffract because the wavelength is factored out of the wave equation ${ }^{15}$. In the present regime, a thermal shock allows us to investigate hitherto untrodden and not previously envisaged ground where light is strongly anti-diffracting and size-dependent, a regime of massy light in which standard beams shrink below the limits of diffractive optics as they evolve.

In summary, we have demonstrated subwavelength nonlinear anti-diffraction in a photorefractive dipolar glass. This occurs when the glass is cast far from equilibrium through a rapid smallamplitude temperature shock, a mechanism known to lead to striking frustration in soft-matter systems ${ }^{15-17}$, and which here leads to a transparent material with enhanced optical transmission properties, including settings for ideal point-like focusing. The beams obey the KGE, so light spots shrink as they propagate, irrespective of their intensity. Using high-aperture optics and super-resolved detection we are able to demonstrate shrinking visible beams down to and below the $1 \mu \mathrm{m}$ scale. In contrast to previously demonstrated subwavelength focusing (see, for example, ref. 18), this occurs for propagation up to several millimetres, with a strong violation of the width/depth-of-focus constraint of diffractive optics. To place our study in context, we note that the laws of optical propagation are also strongly modified in order to lead to anti-diffractive regimes in nanofabricated metamaterials ${ }^{19-23}$, in periodic media ${ }^{24,25}$ and in systems with self-induced transparency ${ }^{26}$. In comparison, our solution does not fundamentally involve wavelength selectivity, directionality, resonances and absorption, and is hence well-suited to imaging applications.

\section{Methods}

From HE to KGE behaviour in nanodisordered ferroelectrics. In nanodisordered ferroelectrics, mesoscopic dipoles (the polar nanoregions, PNRs) give rise to a highly polarizable glass ${ }^{3}$. This glass can then provide optical nonlinearity by coupling to 

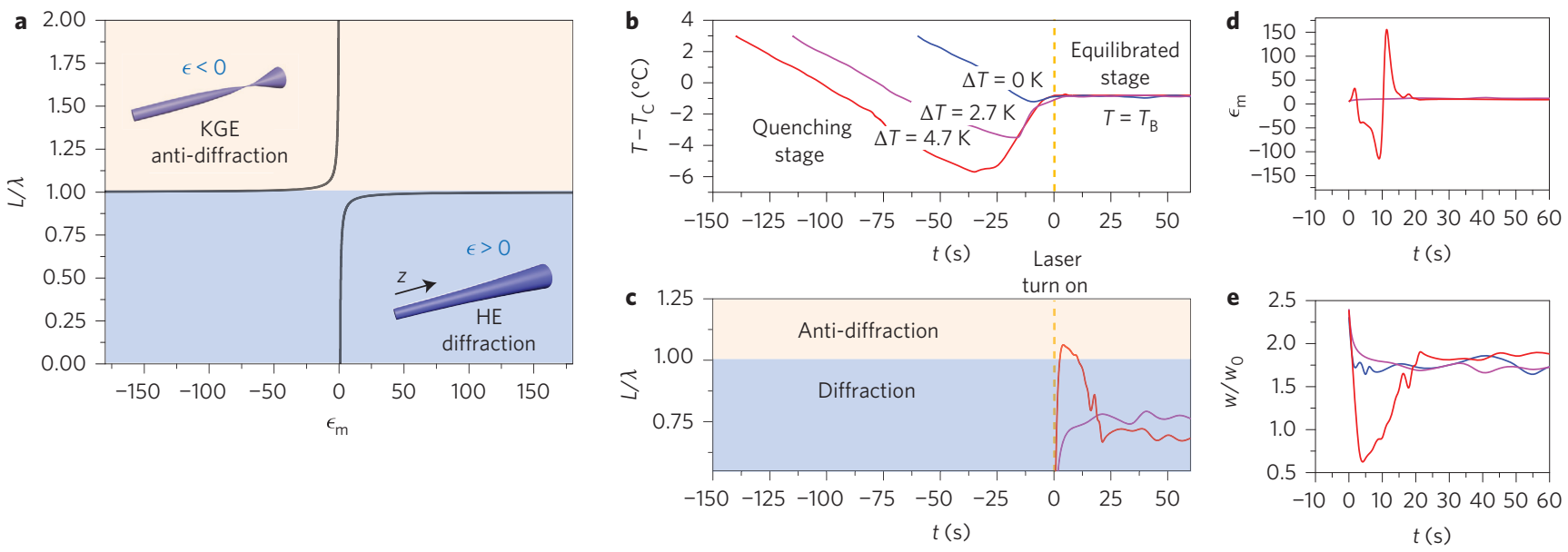

Figure 2 | Accessing the anti-diffraction regime. a, Relationship between the value of $L / \lambda$, the diffraction and anti-diffraction regimes and the effective value of $\epsilon_{\mathrm{m}}$ (see equation (3)). $\mathbf{b}$. Thermal trajectories enacted on the sample with different values of thermal shock, $\Delta T: \Delta T=4.7 \mathrm{~K}$ (red curve), $\Delta T=2.7 \mathrm{~K}$ (purple curve), $\Delta T=0$ (blue curve). c, Values of $L / \lambda$ following the red and purple thermal trajectories as calculated from equation (1). Only the deeper shock (red curve) manifests the $L / \lambda>1$ regime (KGE regime) where anti-diffraction is detected. d, Corresponding values of $\epsilon_{\mathrm{m}}$ as a function of $t$ from equation (3) for the two reported shocks. e, Measured values of output beam width $w$ compared to the input $w_{0}$ after the thermal shock.
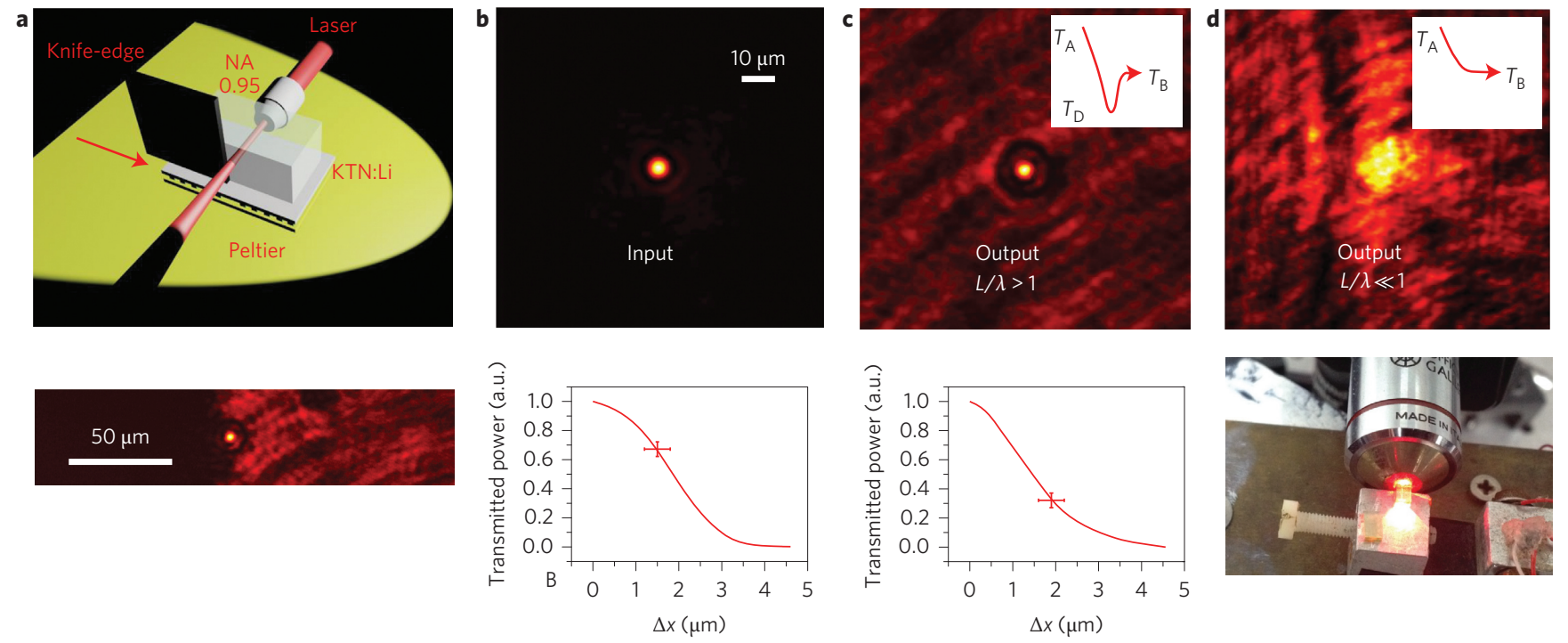

Figure 3 | Micrometre-sized light beams propagating for 134 Rayleigh lengths. a, Schematic of the experimental set-up (top) and image of transmitted light with the knife-edge in the proximity of the output beam (bottom). In the knife-edge technique, a knife-edge is placed directly onto the output crystal facet (at a distance below the diffraction length estimate $\sim 22 \mu \mathrm{m}$ ). The illuminated background in the bottom image is due to the presence of the knife-edge surface (as also in $\mathbf{c}$ ). $\mathbf{b}$, Top: input intensity distribution as collected by the on-axis CCD camera through the collecting lens (NA $\approx 0.35)$. Bottom: highresolution detection of the input beam size using the knife-edge technique, which provides an input width of $1.5 \mu \mathrm{m}$ (see Methods). c, Output beam characterization after an above-threshold thermal shock at the operating temperature (that is, in conditions of $L / \lambda>1$ ). Top: transverse intensity distribution through the on-axis CCD camera. Bottom: knife-edge super-resolved output intensity spot of $1.3 \mu \mathrm{m}$. Note the difference in the shape of the beam detected on the tails, as also indicated by the behaviour in the knife-edge data, compared with the input. Error bars represent the maximum error associated to the knife-edge translation and the measurement of transmitted power. d, Output in conditions of $L / \lambda \ll 1$. Top: output intensity distribution. Bottom: photograph of the crystal, showing how the beam greatly diffracts as it leaves the sample.

light through the diffusion-driven photorefractive response, where light absorbed by impurities diffuses and gives rise to a static electric field $\mathbf{E}_{\mathrm{dc}}=-\left(k_{B} T / q\right) \nabla I / I$, where $k_{\mathrm{B}}$ is the Boltzmann constant, $T$ the crystal temperature, $q$ the elementary charge, and $I=|\mathbf{E}|^{2}$ is the optical intensity ${ }^{27}$. Through the electro-optic response of the PNRs, this gives rise to a scalar change in the index of refraction $n=n_{0}+\Delta n$ (where $\Delta n=-\left(n_{0}^{3} / 2\right) g \epsilon_{0}^{2} \chi_{\mathrm{PNR}}^{2}\left|\mathbf{E}_{\mathrm{d} c}\right|^{2}$; refs 15,28), which causes the HE wave equation to have the form

$$
\nabla^{2} \mathbf{E}-(L / \lambda)^{2}\left(\left.\left.|\nabla| \mathbf{E}\right|^{2}|/ 2| \mathbf{E}\right|^{2}\right)^{2} \mathbf{E}+k^{2} \mathbf{E}=0
$$

Here, $\chi_{\mathrm{PNR}}$ is the PNR low-frequency susceptibility, $g$ is the electro-optic coefficient, $L=4 \pi n_{0}^{2} \epsilon_{0} \sqrt{g} \chi_{\mathrm{PNR}}\left(k_{B} T / q\right)$, and $k=k_{0} n_{0}$ (refs 11,15). To grasp intuitively how this response reproduces the KGE regime typical of massive particles, we can consider localized Gaussian bubbles of light with $E \propto \exp \left(-r^{2} / w_{0}^{2}\right)$, where $w_{0}$ is the spatial extent of the optical excitation and $r^{2}=x^{2}+y^{2}+z^{2}$. The evolution equation reduces to

$$
\nabla^{2} \mathbf{E}\left(1-(L / \lambda)^{2}\right)+\left(k^{2}-(L / \lambda)^{2}\left(6 / w_{0}^{2}\right)\right) \mathbf{E}=0
$$

so for $1<L / \lambda<\left(w_{0} k / \sqrt{6}\right)$ we have a relative change in sign in the terms of the equation, that is, the time-independent $\operatorname{KGE}\left(\nabla^{2}-n_{\mathrm{m}}^{2} k_{0}^{2}\right) \mathbf{E}=0$, with

$$
n_{\mathrm{m}}^{2}(L)=n_{0}^{2}\left(1-(L / \lambda)^{2}\left(6 / k^{2} w_{0}^{2}\right)\right) /\left((L / \lambda)^{2}-1\right)
$$



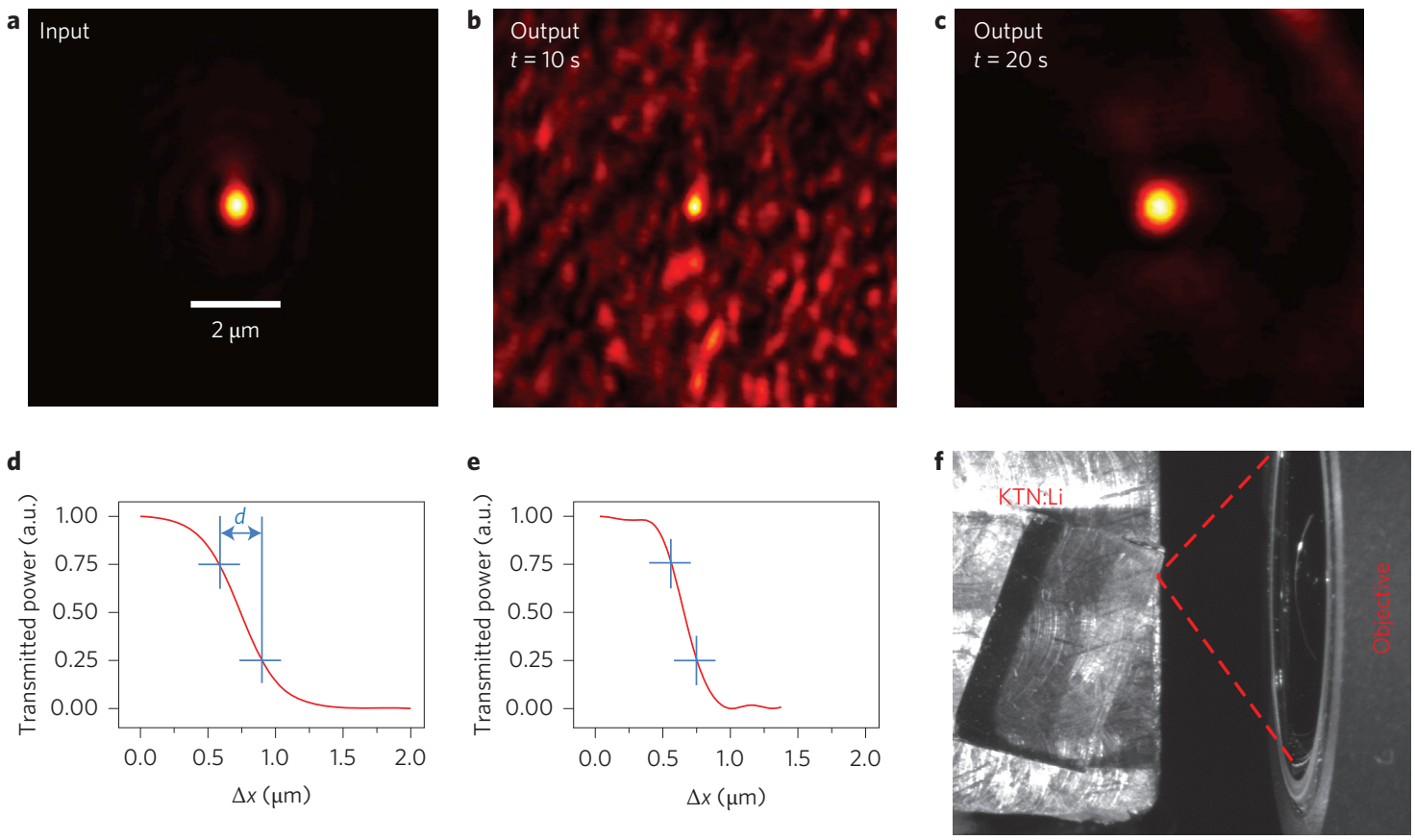

Figure 4 | Reaching below the limits of diffractive optics. a-c, Input beam intensity distribution of width $0.49 \mu \mathrm{m}$ (a), which shrinks to $0.28 \mu \mathrm{m}$ at the output facet (b) and begins to relax once again to a weakly diffracting spot as time passes (c). d,e, Super-resolved knife-edge calibration of the input (d) and output (e) beam. Crosshairs serve to identify the 0.75 and 0.25 transmitted power ratios, so that $w_{0} \simeq \sqrt{2} d$ (see Methods). f, Top-view image of the longworking-distance objective. Note the scattering that accompanies the transient KGE in its initial phases.

For beam propagation along the $z$-axis we can consider Gaussian filaments $E \propto \exp \left(-r_{\perp}^{2} / w_{0}^{2}\right) B(z)$, where $r_{\perp}^{2}=x^{2}+y^{2}$. The wave equation now becomes

$$
-\partial_{z^{\prime} z^{\prime}}^{2}+\nabla_{\perp}^{2} \mathbf{E}-\left((L / \lambda)^{2}-1\right)^{-1}\left(k^{2}-(L / \lambda)^{2}\left(4 / w_{0}^{2}\right)\right) \mathbf{E}=0
$$

where $z^{\prime} \equiv z \sqrt{(L / \lambda)^{2}-1}$. For $1<L / \lambda<\left(w_{0} k / 2\right)$ we have the KGE

$$
\left(\square-n_{\mathrm{m}}^{2} k_{0}^{2}\right) \mathbf{E}=0
$$

with

$$
n_{\mathrm{m}}^{2}(L)=n_{0}^{2}\left(1-(L / \lambda)^{2}\left(4 / k^{2} w_{0}^{2}\right)\right) /\left((L / \lambda)^{2}-1\right)
$$

$\square \equiv-\partial_{z^{\prime} z^{\prime}}^{2}+\nabla_{\perp}^{2}$, time being played by $z^{\prime}$. This approximate treatment also indicates that, for a given $L / \lambda>1$, the KGE will once again be superseded by the HE for beam widths below $w_{0}=(L / \lambda) 2 / k$. The value of $L / \lambda$, through $\chi_{\text {PNR }}$, depends on the operating temperature, on previous thermal history, and is time-dependent. History dependence can be used to program and even partially stabilize material response $\mathrm{e}^{29,30}$ The KGE behaviour is activated without affecting the values of material dielectric constant $\epsilon$ (and $\mu$ ), without nanostructuring, or absorption, and consistently through the small nonlinear optical response regime (that is, the maximum local value of index modulation is $\Delta n \ll n_{0}\left(\Delta n / n_{0} \approx 10^{-4} \text { for } w_{0} \approx 10 \mu \mathrm{m}\right)^{11,12}$.

Anti-diffraction experiments: optical set-up. Experiments were carried out in lithium-enriched potassium-tantalate-niobate (KTN:Li), an evolution of ferroelectric KLTN (potassium-lithium-tantalate-niobate) ${ }^{31}$. Strong out-of-equilibrium behaviour analogous to that of relaxor ferroelectrics was observed in our specific composition $\mathrm{K}_{1-x} \mathrm{Ta}_{1-y} \mathrm{Nb}_{y} \mathrm{O}_{3}: \mathrm{Li}_{x}$ with $x=0.003$ and $y=0.36$, giving rise to a glass-forming liquid dielectric $^{32}$. Photorefraction was mediated by $\mathrm{Cu}$ impurities (approximately 0.001 atoms per mole). A continuous-wave beam, either from a He-Ne laser $(\lambda=632.8 \mathrm{~nm})$ or from a doubled Nd:YAG $(\lambda=532 \mathrm{~nm}$ laser), was first expanded and then focused to a spot with an intensity full-width at half-maximum of $\Delta x=\Delta y \approx 13 \mu \mathrm{m}$ (for 632.8 $\mathrm{nm}$ ) at the input face of the zero-cut $2.6 \times 3.0 \times 6.0 \mathrm{~mm}$ sample (with $n_{0}=2.2$ ). The beam, polarized in the $x$-direction, was made to propagate inside the crystal for a distance $L_{z} \approx 3.0 \mathrm{~mm}$. The crystal was rotated to an arbitrary angle $\theta$ in the $x-z$ plane. The output intensity distribution of the beam was captured by a charge-coupled device $(\mathrm{CCD})$ camera through an imaging lens $(\mathrm{NA} \approx 0.35)$. Light scattered by the dipolar glass was collected by a second CCD camera placed above the sample in the $y$-direction through a high-aperture microscope $(\mathrm{NA} \approx 0.8)$ positioned so as to image the plane of propagation in the sample. At visible wavelengths, the crystal absorption coefficient was $\alpha \approx 2 \mathrm{~cm}^{-1}$

Thermal shocks. We activated a strong dielectric response using non-monotonic temperature trajectories $T(t)$ (refs 15,33). This required the sample to be operated in its non-ergodic phase ${ }^{3}$. This phase was identified through dielectric constant measurements in the proximity of the room-temperature Curie point $T_{\mathrm{C}}=14.5^{\circ}$ (see Supplementary Information). In this phase, a temperature controller was used to drive the current of a Peltier junction placed directly below the crystal in the $y$-direction, taking the sample through a thermal shock $T(t)$ before the light was switched on: the sample was cooled from an initial temperature $T_{\mathrm{A}}$ to the final operating temperature $T_{\mathrm{B}}<T_{\mathrm{A}}$, passing through a cooling stage at a lower temperature $T_{\mathrm{D}}<\left(T_{\mathrm{A}}, T_{\mathrm{B}}\right)$, where $\Delta T=T_{\mathrm{B}}-T_{\mathrm{D}}$ is the shock amplitude (Supplementary Fig. 3). The shock led to a transient anomalous response, activating the $L>\lambda$ regime (that is, elevated values of $\chi_{\mathrm{PNR}}$ ) even though $T_{\mathrm{A}}$ and $T_{\mathrm{B}}$ differed by only a few degrees.

Super-resolution knife-edge measurements. The non-paraxial anti-diffraction experiments were carried out by launching the laser beam directly into an NA $\approx 0.8$ (used without oil) microscope objective, as shown in Fig. $3 \mathrm{~d}$ and represented in Fig. 3a, where the knife-edge scheme is illustrated, and using a dry long-distance objective (Fig. 4f). We mounted a plate on a three-axis nanopositioning stage, aligned it to the plane orthogonal to the laser propagation axis $Z$, and placed it in the proximity of the output plane of the objective, with a precision below $1 \mu \mathrm{m}$ (this is required because the confocal parameter of the beams is on the order of tens of micrometres). The power of the transmitted light as a function of the lateral $x$ displacement of the plate edge $\Delta x$ was then detected using a silicon-head power meter. The beam width was deduced from the transmitted power plot (Figs $3 b, c$ and $4 \mathrm{~d}, \mathrm{e}$ ) by fitting the data with what is expected for a two-dimensional Gaussian beam intensity shape of width $w_{0}$. Specifically, the transmitted power $P_{\text {out }}$ normalized to the maximum transmission $P_{\max }$ follows the law $P(\Delta x)_{\text {out }} / P_{\max }=$ $(1 / 2)\left(1-\operatorname{erf}\left(\sqrt{2} \Delta x / w_{0}\right)\right)$, so that the displacement $d$ that brings the normalized fraction from 0.75 to 0.25 quantifies the beam width $w_{0} \simeq \sqrt{2} d$ (see cross hairs in Fig. 4 d,e). For the output of Fig. 3c, the launch objective was placed at the input facet of the sample and the same technique was used with the plate finely aligned with the output facet. A specific stage of the knife-edge measurement is reported in the bottom panel of Fig. 3a (note the dark region to the left of the ultra-focused beam). The background detected in the output image of Fig. $3 \mathrm{c}$ is due to the residual on-axis scattering that enters the imaging lens after a double-reflection from the knife plate and from the input facet of the sample.

Received 7 November 2014; accepted 20 January 2015; published online 2 March 2015

\section{References}

1. Hell, S. W., Schmidt, R. \& Enger, A. Diffraction-unlimited three-dimensional optical nanoscopy with opposing lenses. Nature Photon. 3, 381-387 (2009).

2. Kawata, S., Inouye, Y. \& Verma, P. Plasmonics for near-field nano-imaging and superlensing. Nature Photon. 3, 388-394 (2009). 
3. Bokov, A. A. \& Ye, Z.-G. Dielectric relaxation in relaxor ferroelectrics. J. Adv. Dielectrics 2, 1241010 (2012).

4. Shvartsman, V. V. \& Lupascu, D. C. Lead-free relaxor ferroelectrics. J. Am. Ceram. Soc. 95, 1-26 (2012).

5. Trillo, S. \& Torruellas, W. Spatial Solitons (Springer-Verlag, 2001).

6. Boardman, A. D. \& Sukhorukov, A. P. Soliton Driven Photonics (Kluwer Academic, 2001).

7. Kivshar, Y. S. \& Agrawal, G. P. Optical Solitons (Academic Press, 2003).

8. Chen, Z., Segev, M. \& Christodoulides, D. N. Optical spatial solitons: historical overview and recent advances. Rep. Prog. Phys. 75, 086401 (2012).

9. Mandl, F. Quantum Field theory (Wiley, 1984)

10. Born, M. \& Wolf, E. Principles of Optics 7th edn (Cambridge Univ. Press, 1999).

11. Conti, C., Agranat, A. J. \& DelRe, E. Subwavelength optical spatial solitons and three-dimensional localization in disordered ferroelectrics: towards metamaterials of nonlinear origin. Phys. Rev. A 84, 043809 (2011).

12. Yariv, A. Quantum Electronics (Wiley, 1988).

13. Verdeyen, J. T. Laser Electronics (Prentice Hall, 1981).

14. Kovacs, A. J. Glass transition in amorphous polymers a phenomenological study. Adv. Polym. Sci. 3, 394-508 (1963).

15. DelRe, E., Spinozzi, E., Agranat, A. J. \& Conti, C. Scale-free optics and diffractionless waves in nanodisordered ferroelectrics. Nature Photon. 5, 39-42 (2011).

16. Mossa, S. \& Sciortino, F. Crossover (or Kovacs) effect in an aging molecular liquid. Phys. Rev. Lett. 92, 045504 (2004).

17. Leuzzi, L. \& Nieuwenhuizen, T. M. Thermodynamics of the Glassy State (Taylor \& Francis, 2008).

18. Park, J. H. et al. Subwavelength light focusing using random nanoparticles. Nature Photon. 7, 454-458 (2013).

19. Veselago, V. G. The electrodynamics of substances with simultaneously negative values of $\varepsilon$ and $\mu$. Sov. Phys. Usp. 10, 509-514 (1968).

20. Pendry, J. B. Negative refraction makes a perfect lens. Phys. Rev. Lett. 85, 3966-3969 (2000).

21. Fang, N., Lee, H., Sun, C. \& Zhang, X. Sub-diffraction-limited optical imaging with a silver superlens. Science 308, 534-537 (2005).

22. Shalaev, V. M. Optical negative-index metamaterials. Nature Photon. 1, 41-48 (2007)

23. Powell, D. A., Shadrivov, I. V. \& Kivshar, Y. S. Nonlinear electric metamaterials Appl. Phys. Lett. 95, 084102 (2009).

24. Kosaka, H. et al. Self-collimating phenomena in photonic crystals. Appl. Phys. Lett. 74, 1212-1214 (1999).

25. Eisenberg, H. S., Silberberg, Y., Morandotti, R. \& Aitchison, J. S. Diffraction management. Phys. Rev. Lett. 85, 1863-1866 (2000).
26. Firstenberg, O., London, P., Shuker, A., Ron, A. \& Davidson, N. Elimination, reversal and directional bias of optical diffraction. Nature Phys. 5, 665-668 (2009).

27. Crosignani, B., Degasperis, A., DelRe, E., Di Porto, P. \& Agranat, A. J. Nonlinear optical diffraction effects and solitons due to anisotropic charge-diffusion-based self-interaction. Phys. Rev. Lett. 82, 1664-1667 (1999).

28. DelRe, E. \& Conti, C. in Nonlinear Photonics and Novel Optical Phenomena Vol. 170 (eds Chen, Z. \& Morandotti, R.) Ch. 8 (Springer-Verlag, 2012).

29. Parravicini, J., Conti, C., Agranat, A. J. \& DelRe, E. Programming scale-free optics in disordered ferroelectrics. Opt. Lett. 37, 2355-2357 (2012).

30. Parravicini, J., Conti, C., Agranat, A. J. \& DelRe, E. Rejuvenation in scale-free optics and enhanced diffraction cancellation life-time. Opt. Express 20, 27382-27387 (2012)

31. Agranat, A., Hofmeister, R. \& Yariv, A. Characterization of a new photorefractive material: $\mathrm{K}_{1-y} \mathrm{~L}_{y} \mathrm{~T}_{1-x} \mathrm{~N}_{x}$. Opt. Lett. 17, 713-715 (1992).

32. Ben Ishai, P., Agranat, A. J. \& Feldman, Y. Confinement kinetics in a KTN:Cu crystal: experiment and theory. Phys. Rev. B 73, 104104 (2006).

33. Alberici-Kious, F., Bouchaud, J. P., Cugliandolo, L. F., Doussineau, P. \& Levelut, A. Aging in $\mathrm{K}_{1-x} \mathrm{Li}_{x} \mathrm{TaO}_{3}$ : a domain growth interpretation. Phys. Rev. Lett. 81, 4987-4990 (1998).

\section{Acknowledgements}

The research leading to these results was supported by funding from the Italian Ministry of Research (MIUR) through the 'Futuro in Ricerca' FIRB grant PHOCOS-RBFR08E7VA and from the European Research Council under the European Community Seventh Framework Programme (FP7/2007-2013)/ERC grant agreement no. 201766. Partial funding was received through the SMARTCONFOCAL project of the Regione Lazio and through the PRIN 2012BFNWZ2 and Sapienza 2013PHOTOANDERSON projects. A.J.A. acknowledges support from the Peter Brojde Center for Innovative Engineering.

\section{Author contributions}

E.D. and C.C. conceived and developed the experiments and theory. R.A. designed and fabricated the KTN:Li samples and participated in the analysis and interpretation of results. F.D. and J.P. carried out the experiments and data analysis. G.P. carried out the dielectric characterization of the material.

\section{Additional information}

Supplementary information is available in the online version of the paper. Reprints and permissions information is available online at www.nature.com/reprints. Correspondence and requests for materials should be addressed to E.D.

\section{Competing financial interests}

The authors declare no competing financial interests. 